\title{
STABILITY OF SHALLOW MIXING LAYERS WITH ASYMMETRIC BASE FLOW PROFILE
}

\author{
Vladislav Kremenetsky, Andrei Kolyshkin \\ Riga Technical University, Latvia \\ vlad@sal.lv, andrejs.koliskins@rtu.lv
}

\begin{abstract}
Linear stability of shallow flows is usually analyzed in the literature under the assumption that the base flow profile is symmetric with respect to the transverse coordinate. Another widely used assumption is the rigid-lid assumption where perturbations at the upper surface are not considered. Experimental data show that the symmetry of the base flow can be distorted by a non-uniform friction in the transverse direction of the flow. Such a situation occurs in applications in case of the presence of aquatic vegetation (for example, in case of floods). In this case there is a sharp change of the resistance force at the interface. Experiments show that nonuniform resistance force plays an important role in development of the mixing layer. In the present paper linear stability analysis of shallow mixing layers with non-uniform friction is investigated. Both previously mentioned assumptions are removed and the problem is solved for asymmetric base flow profile for arbitrary Froude numbers. The friction coefficient is assumed to be a function of the transverse coordinate. Experimentally measured asymmetric base flow profile is used in the paper. The linear stability problem is solved numerically by a collocation method based on Chebyshev polynomials using different values of the parameters of the problem.
\end{abstract}

Keywords: shallow flows, linear stability, asymmetric base flow.

\section{Introduction}

Shallow flows often occur in applications (examples include flows at river junctions or in compound channels). Linear stability of such flows is usually analyzed under the assumption that the base flow is symmetric with respect to the transverse coordinate [1-4]. Experimental data [5] show that symmetry of the base flow can be distorted by the presence of the non-uniform friction force acting in the transverse direction. Such a situation often occurs during floods where moving fluid is in contact with aquatic vegetation.

Linear stability of shallow mixing layers for a symmetric base flow (a hyperbolic tangent velocity profile) is analyzed in [6;7], where it is shown that a non-uniform friction stabilizes the flow. In the present paper linear stability of shallow mixing layers with non-uniform friction is investigated under the assumption that the base flow is asymmetric with respect to the transverse coordinate. The base flow profile is obtained from experimental data [5] where semi-analytical formulas for the velocity distribution in the transverse direction are also obtained. Linear stability is analyzed for several experimental cases quoted in [5]. Spline interpolation is used to smooth experimental velocity profiles. As it is shown in [5], the velocity distribution has a two-layer structure where sharp change of the velocity occurs in a shear layer at the boundary between aquatic vegetation and the main channel. The presence of an inflection point in the velocity profile indicates possible hydrodynamic instabilities. Linear stability analysis is used in the paper to calculate the critical values of the friction coefficient in the main channel. Results of numerical calculations are discussed.

\section{Base flow and linearized equations}

Consider the following dimensionless shallow water equations [4]:

$$
\left\{\begin{array}{c}
\frac{\partial h}{\partial t}+\frac{\partial}{\partial x}(u h)+\frac{\partial}{d y}(v h)=0 \\
\frac{\partial u}{\partial t}+u \frac{\partial u}{\partial x}+v \frac{\partial u}{\partial y}+\frac{\partial h}{\partial x}-S_{O x}+c_{f} \frac{u \sqrt{u^{2}+v^{2}}}{h}=0 \\
\frac{\partial v}{\partial t}+u \frac{\partial v}{\partial x}+v \frac{\partial v}{\partial y}+\frac{\partial h}{\partial y}-S_{O y}+c_{f} \frac{v \sqrt{u^{2}+v^{2}}}{h}=0
\end{array},\right.
$$

obtained from common shallow water equations using values $H, H / \sqrt{g H}, \sqrt{g H}$ as scales of length, time and velocity respectively; 
$g$ - the gravitational acceleration;

$H$ - the maximum depth of the channel;

$c_{f}$ - the friction coefficient;

$S_{O y}$ - the bed slope of the channel along $x$;

$S_{O y}-$ the bed slope of the channel along $y$.

The dimensionless base flow is assumed to be of the form

$$
u=(U(y), 0), h=H(y) .
$$

It follows from the shallow water equations that flow (2) exists if the following conditions are satisfied ( $S_{f x}$ is the $x$-component of the bed-friction force):

$$
\frac{d H}{d y}-S_{O y}=0 ; S_{O x}-S_{f x}=0 .
$$

If the undisturbed water depth $H$ is constant, then the base flow (2) exists under the condition

$$
S_{O x}=\frac{c_{f} U^{2}}{H}=c_{f} F r^{2},
$$

where $F r=U / \sqrt{H}-$ the local Froude number of the flow.

Since the bed slope is constant, condition (4) implies that the product of the friction coefficient and the local Froude number must be constant at any point in the transverse direction. Suppose that there is a layer of aquatic vegetation in the interval $y>0$ ( $y$ is the transverse coordinate) where the friction coefficient $c_{v e g}$ is considerably larger than in the main channel $\left(c_{m c}\right)$. Since $\mathrm{H}$ is assumed to be constant in sequel, condition (4) can be written in the form

$$
c_{m c} F r_{m c}^{2}=c_{v e g} F r_{v e g}^{2} \text {, }
$$

The subscripts " $m c$ " and "veg" correspond to the main channel and vegetated area, respectively.

We assume that the friction coefficient has the form

$$
c_{f}=\frac{c_{m c}+c_{v e g}}{2}+\frac{c_{m c}-c_{v e g}}{2} \tanh (\alpha y) .
$$

The base flow velocity profile used in our paper is based on the model developed in [5]. To determine the base flow profile we use the experimental data from Table 1 in [5] and semi-analytical expressions obtained in [5].

The flow consists of four regions:

1) the flow in the main channel with constant velocity $U_{2}$;

2 ) the outer layer of the width $\delta_{O}$ with the velocity

$$
U_{O}=U_{2}\left[3 \tanh ^{2}\left(\sqrt{\frac{3}{4\left(U_{m} / U_{2}+2\right)}} \frac{\left(y-y_{m}\right)}{\delta_{O}}+C\right)-2\right]
$$

where

$$
C=\operatorname{arctanh} \sqrt{1+\frac{U_{m} / U_{2}-1}{3}} ;
$$

3) the inner layer of the width $\delta_{I}$ with the velocity

$$
U_{I}=U_{1}+U_{S}\left(1+\operatorname{th}\left(\frac{y-y_{O}}{\delta_{I}}\right)\right)
$$

4) the flow in the vegetated area with constant velocity $U_{1}$. 
Here $U_{S}=U\left(y_{O}\right)-U_{1}$ is the slip velocity, $y_{O}$ is the inflection point of the velocity profile, the width of the inner layer $\delta_{I}=0.5\left(C_{D} \alpha\right)^{-1}$, where $C_{D} \alpha$ is the vegetation drag.

The flows in the inner and outer layers match at the point $y_{m}$. Due to experimental errors exact matching is not possible. In order to smooth the velocity profile we approximated the base flow velocity and its derivative by cubic splines. The obtained dimensionless velocity profiles (the scale of velocity in this case is $\sqrt{g h}$, where $h$ is the depth of the flow) are shown on Fig. 1. The curves in Fig. 1 correspond to the following experimental conditions (see Table 1 in [5]): 1 - Case 1, 2 - Case 4, 3 - Case 7, 4-Case 9,5-Case 11. The two-layer structure is clearly seen from the graphs. The presence of the inflection point in the velocity profiles points to hydrodynamic instability of the base flow.

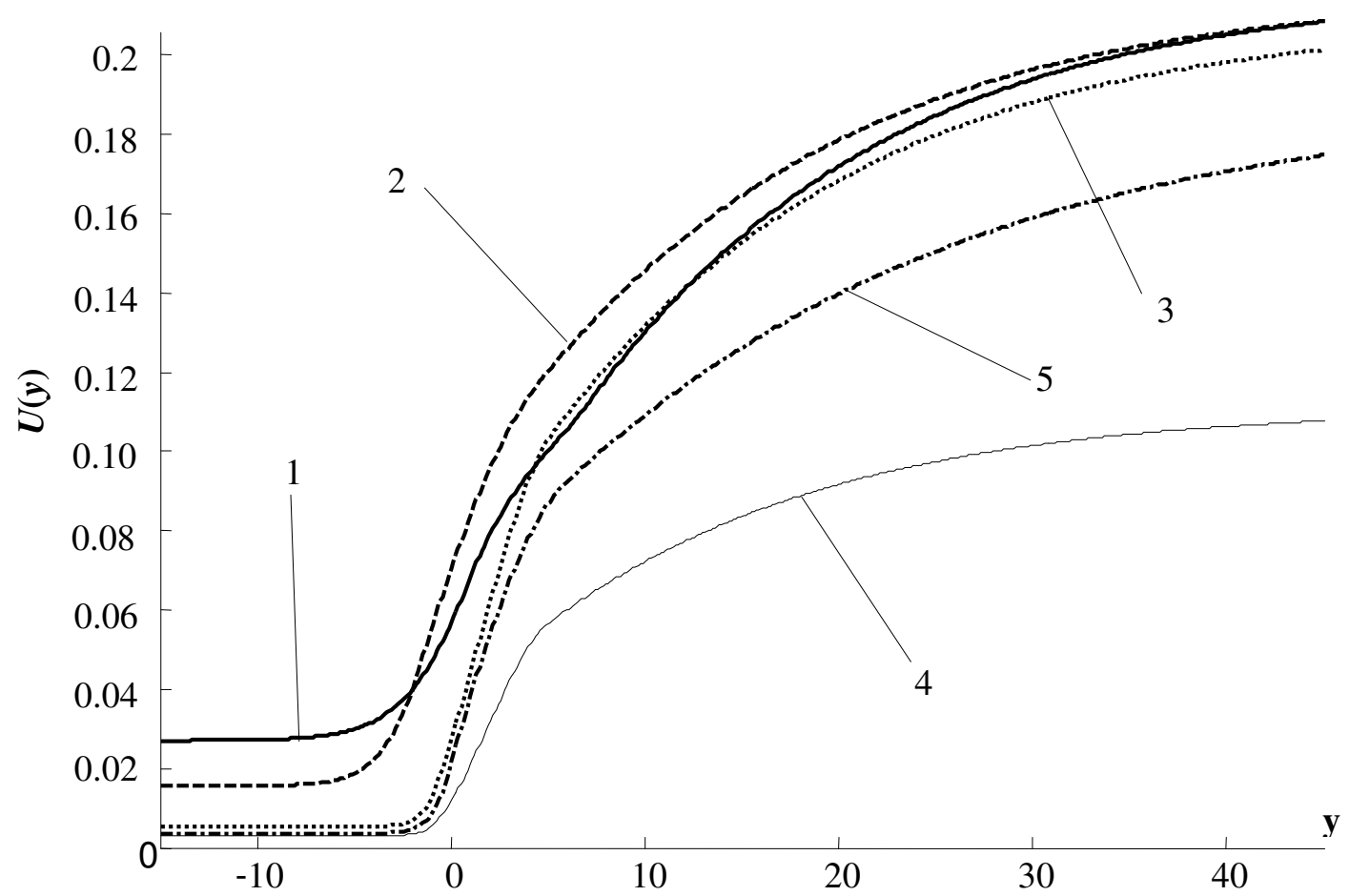

Fig. 1. Base flow velocity distribution for five experimental cases in [5]:

1-Case 1;2-Case 4;3-Case 7; 4 - Case 9; 5 - Case 11

Using the method of normal modes we assume perturbations of the form

$$
\begin{aligned}
& u=U(y)+u^{\prime}(y) e^{-\lambda t+i k x} \\
& v=v^{\prime}(y) e^{-\lambda t+i k x} \\
& h=1+h^{\prime}(y) e^{-\lambda t+i k x}
\end{aligned}
$$

where $u^{\prime}, v^{\prime}$ and $h^{\prime}$ are small perturbations.

Substituting (7) into (1) and linearizing the equations in the neighborhood of the base flow (2) we obtain the following linear system for the perturbed quantities:

$$
\begin{aligned}
& H \frac{d v^{\prime}}{d y}+i k u^{\prime} H+i k h^{\prime} U-\lambda h^{\prime}=0 \\
& \frac{d h^{\prime}}{d y}+\frac{c_{f} F r^{2} v^{\prime}}{U}+i k v^{\prime} U-\lambda v^{\prime}=0 \\
& u^{\prime}\left(i k U+\frac{2 c_{f} F r^{2}}{U}\right)+v^{\prime} \frac{d U}{d y}+h^{\prime}\left(i k-\frac{c_{f} F r^{2}}{h}\right)-\lambda u^{\prime}=0
\end{aligned} .
$$


The boundary conditions have the form (boundary conditions for $u^{\prime}$ and $h^{\prime}$ are not needed because the problem is inviscid):

$$
v^{\prime}( \pm \infty)=0
$$

The eigenvalues $\lambda$ of the boundary value problem (8), (9) determine linear stability of the base flow (2). If all real parts of $\lambda$ are positive, then the base flow is linearly stable. If at least one of the eigenvalues $\lambda$ has a negative real part, the base flow is linearly unstable.

\section{Numerical method}

The problem (8)-(9) is solved by means of the Chebyshev collocation method. Using the substitution $\xi=2 / \pi$ arctany we map the interval $-\infty<y<+\infty$ onto the interval $-1<\xi<1$. In terms of the transformed variable the solution is sought in the form:

$$
\begin{aligned}
& u^{\prime}(\xi)=\sum_{i=0}^{N} a_{i} T_{i}(\xi) \\
& v^{\prime}(\xi)=\sum_{i=0}^{N} b_{i}\left(1-\xi^{2}\right) T_{i}(\xi) \\
& h^{\prime}(\xi)=\sum_{i=0}^{N} c_{i} T_{i}(\xi)
\end{aligned}
$$

where $T_{i}(\xi)=\cos (i \arccos \xi)$ is the Chebyshev polynomial of the first kind of order $i$.

The collocation points are

$$
\xi_{j}=\cos \frac{\pi j}{N}, j=0,1, \ldots, N
$$

The parameters used to determine the base flow are shown in Table 1. The results of linear stability calculations are shown in the next section.

\section{Experimental data from [5]}

Table 1

\begin{tabular}{|c|c|c|c|c|c|}
\hline \multirow{2}{*}{ Parameters } & \multicolumn{5}{|c|}{ Cases } \\
\cline { 2 - 6 } & $\mathbf{1}$ & $\mathbf{4}$ & $\mathbf{7}$ & $\mathbf{9}$ & $\mathbf{1 1}$ \\
\hline$C_{D} \alpha, \mathrm{cm}^{-1}$ & 0.092 & 0.285 & 2.43 & 2.04 & 2.43 \\
\hline$h, \mathrm{~cm}$ & 6.8 & 6.6 & 6.6 & 6.8 & 13.9 \\
\hline$y_{O}, \mathrm{~cm}$ & 1.34 & -0.65 & 0.48 & 0.51 & 0.81 \\
\hline$y_{m}, \mathrm{~cm}$ & 4.12 & 1.54 & 2.21 & 2.01 & 2.84 \\
\hline$\delta_{I}, \mathrm{~cm}$ & 3.71 & 2.61 & 1.24 & 1.06 & 1.35 \\
\hline$\delta_{O}, \mathrm{~cm}$ & 15.95 & 16.69 & 16.50 & 15.20 & 21.54 \\
\hline$U_{1}, \mathrm{~cm} \cdot \mathrm{s}^{-1}$ & 2.21 & 1.25 & 0.43 & 0.25 & 0.41 \\
\hline$U_{2}, \mathrm{~cm} \cdot \mathrm{s}^{-1}$ & 17.68 & 17.37 & 16.82 & 9.05 & 22.02 \\
\hline$U_{S}, \mathrm{~cm} \cdot \mathrm{s}^{-1}$ & 3.68 & 3.72 & 3.41 & 1.79 & 4.51 \\
\hline$U_{m}, \mathrm{~cm} \cdot \mathrm{s}^{-1}$ & 7.41 & 7.87 & 6.71 & 3.64 & 9.00 \\
\hline
\end{tabular}

\section{Results and discussion}

Examples of marginal stability curves are shown in Figs. 2 and 3. The flow is stable above the curves and unstable below the curves. The maximum of the possible values of $c_{m c}$ represents the critical value of the friction coefficient in the main channel: $c_{c r}=\max _{k} c_{m c}$. The critical values $c_{c r}$ are calculated for all five cases shown in Table 1. The results are summarized in Table 2. As can be seen from Table 2, the critical values of the friction coefficient seem to be independent on the Froude number (however, the Froude numbers used in the experiments in [5] do not exceed 0.22 so that it is not clear whether this conclusion holds for other values of the Froude number). It should be also pointed out that the base flow used in the paper is obtained from averaged long-term evolution of the flow, so that it includes nonlinear effects and changes in the initial structure of the mixing layer. However, such an approach is used earlier in the literature [8] and found to be useful in characterizing the development of coherent structures (at least at the initial stage). More detailed parametric study is 
needed in order to fully assess the effect of asymmetry of the base flow on the stability characteristics of shallow mixing layers.

Table 2

Critical values of the friction coefficient in the main channel

\begin{tabular}{|c|c|c|c|c|c|}
\hline \multirow{2}{*}{ Parameters } & \multicolumn{5}{|c|}{ Cases } \\
\cline { 2 - 6 } & $\mathbf{1}$ & $\mathbf{4}$ & $\mathbf{7}$ & $\mathbf{9}$ & $\mathbf{1 1}$ \\
\hline$c_{c r}$ & 0.031 & 0.017 & 0.018 & 0.020 & 0.038 \\
\hline$F r_{m c}$ & 0.22 & 0.22 & 0.21 & 0.11 & 0.19 \\
\hline
\end{tabular}

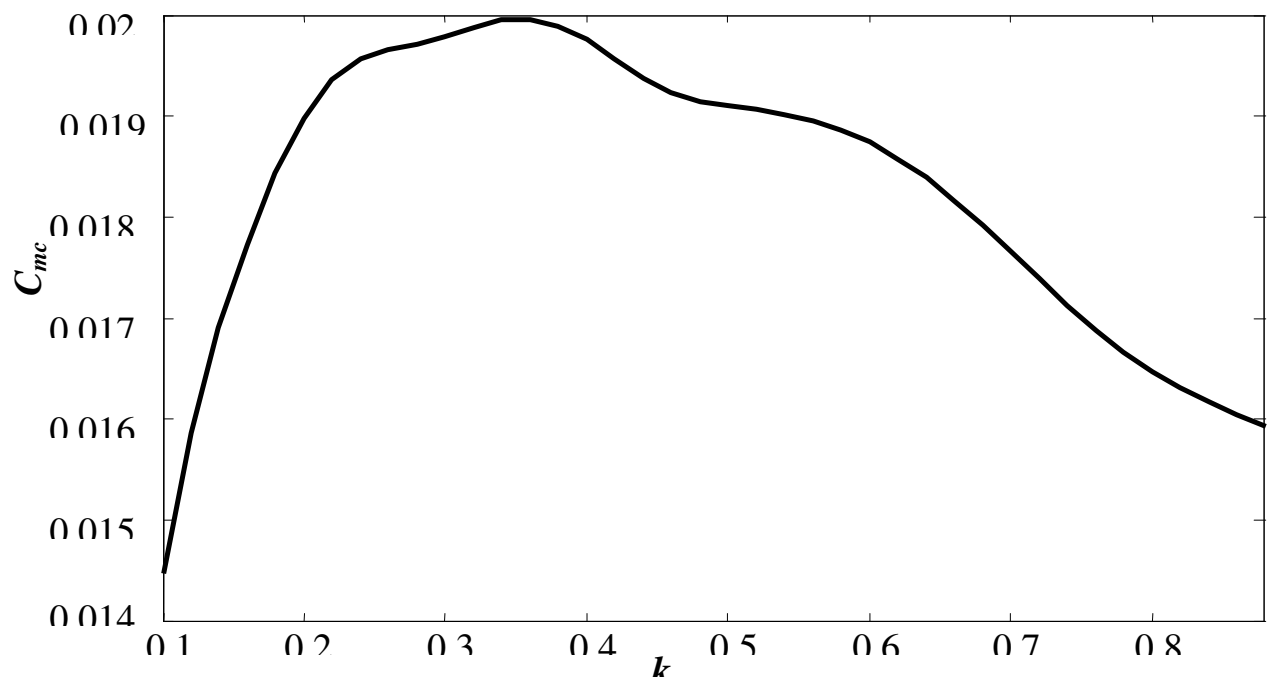

Fig. 2. Marginal stability curve for Case 9

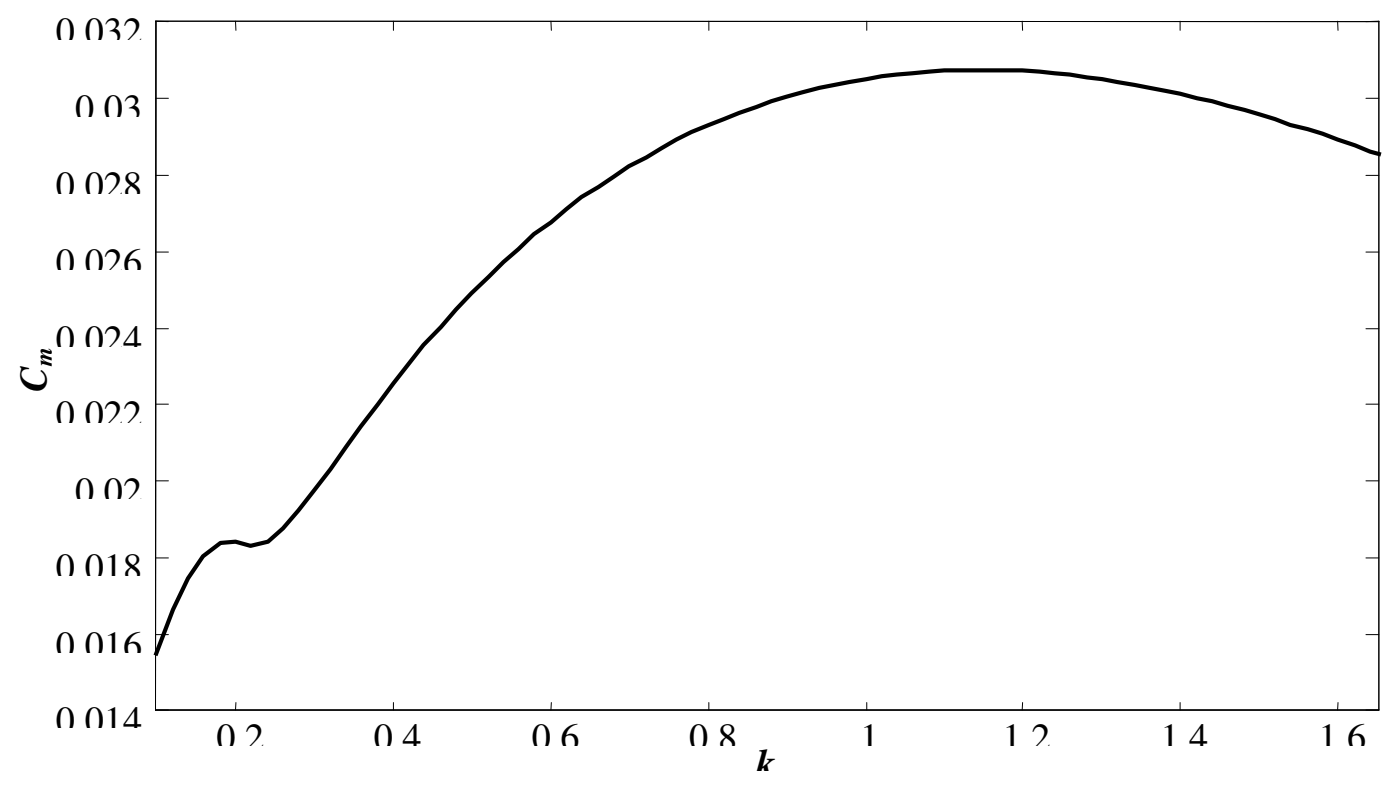

Fig. 3. Marginal stability curve for Case 1

\section{Conclusions}

Linear stability of asymmetric base flow profile is investigated in the present paper. The asymmetry of the base flow is caused by the presence of an aquatic vegetated layer where the resistance force is much larger than in the main channel. The critical values of the friction coefficient are independent on the Froude number (at least in the range $0<F r<0.22$ used in the experiments). 


\section{Acknowledgement}

This work was partially supported by the grant 623/2014 of the Latvian Council of Science.

\section{References}

1. Chu V.H., Wu J.H., Khayat R.E. Stability of transverse shear flows in shallow channel. Journal of Hydraulic Engineering, vol. 117, 1991, pp. 1370-1388.

2. Chen D., Jirka G.H. Linear stability analysis of turbulent mixing layers and jets in shallow water layers. Journal of Hydraulic Research, vol. 136, 1998, pp. 815-830.

3. Ghidaoui M.S., Kolyshkin A.A. Linear stability analysis of lateral motions in compound open channels. Journal of Hydraulic Engineering, vol. 125, 1999, pp. 871-880.

4. Ghidaoui M.S., Kolyshkin A.A. Gravitational and shear instabilities in compound and composite channels. Journal of Hydraulic Engineering, vol. 128, 2002, pp. 1076-1086.

5. White B.L., Nepf H.M. A vortex-based model of velocity and shear stress in a partially vegetated shallow channel. Water Resources Research, vol. 44, 2008, 15 p. (paper W01412).

6. Kolyshkin A., Kremenetsky V. Stability of shallow mixing layers with free surface and nonuniform friction. Proceedings of the $15^{\text {th }}$ conference on applied mathematics (APLIMAT), February 2-4, 2016, Bratislava, Slovakia, pp. 674-679.

7. Kolyshkin A., Kremenetsky V. Stability of shallow flows: a weakly nonlinear approach. AIP Conference Proceedings, vol. 1798, 2017, Article number 020081.

8. Van Prooijen B. C., Uijttewaal W.S.J. A linear approach for the evolution of coherent structures in shallow mixing layers. Physics of Fluids, vol. 14, 2002, pp. 4105-4114. 